\title{
Nutritional benefit of laparoscopic jejunostomy during neoadjuvant chemotherapy for obstructing esophageal cancer
}

\author{
KEN NAGATA, HIRONORI TSUJIMOTO, HIROMI NAGATA, MANABU HARADA, \\ NOZOMI ITO, SHINSUKE NOMURA, HIROYUKI HORIGUCHI, SHUICHI HIRAKI, \\ SUEFUMI AOSASA, KAZUO HASE and HIDEKI UENO
}

Department of Surgery, National Defense Medical College, Tokorozawa, Saitama 359-8513, Japan

Received August 29,2018; Accepted September 4, 2019

DOI: $10.3892 /$ mco.2019.1938

\begin{abstract}
Neoadjuvant chemotherapy (NAC) confers a survival benefit in esophageal carcinoma, but it is difficult to perform in patients who cannot receive enteral feeding due to an esophageal obstruction. In the current study, the nutritional benefit of laparoscopic jejunostomy (Lap-J) was evaluated in patients with NAC for obstructing esophageal cancer. A total of 91 patients with esophageal cancer who received NAC between 2009 and 2017 were included in the present study. Lap-J was performed prior to NAC in 15 patients (16.5\%) with an obstructing tumor. Patients with NAC without Lap-J were used as the control group $(n=76)$. Nutritional parameters and surgical outcomes of the two groups were compared retrospectively. In the patients with Lap-J, 14 of the 15 patients $(93.3 \%)$ did not experience any procedure-associated complications. No mortalities were associated with Lap-J. Significant decreases in total serum protein, albumin, hemoglobin concentrations and prognostic nutritional index (PNI) occurred following NAC in the control but not in the Lap-J group. Serum albumin and the improved modified Glasgow prognostic score increased significantly after NAC in the Lap-J group but not in the control group. In conclusion, perioperative nutritional support with Lap-J was safe and effective in patients with NAC for obstructing esophageal cancer.
\end{abstract}

\section{Introduction}

Neoadjuvant chemotherapy (NAC) followed by surgery is a standard treatment of resectable stage II or III esophageal squamous cell carcinoma in Japan. A prospective randomized trial comparing postoperative adjuvant chemotherapy with cisplatin and 5-fluorouracil $(\mathrm{CF})$ regimen vs. preoperative chemotherapy

Correspondence to: Professor Hironori Tsujimoto, Department of Surgery, National Defense Medical College, 3-2 Namiki, Tokorozawa, Saitama 359-8513, Japan

E-mail: tsujihi@ndmc.ac.jp

Key words: laparoscopic jejunostomy, minimal invasive surgery, esophageal cancer, enteral nutrition
(JCOG9907), showed a significant survival advantage of NAC (1). However, significant weight loss and malnutrition may occur during NAC, which usually has a number of weeks duration (2), and are of concern especially in patients with tumors that obstruct passage of liquids and solid food. Treatment of patients with obstructing tumors may be restricted to surgery without NAC, or with NAC with inadequate enteral nutrition. Laparoscopic jejunostomy (Lap-J) is easy to perform and safely provides nutritional support of patients with cervical and esophageal obstructing tumors and those with advanced gastric cancer (3). This study evaluated the nutritional benefit of Lap-J in patients receiving NAC with a CF regimen for obstructing, locally advanced esophageal cancer.

\section{Materials and methods}

This study retrospectively evaluated 91 patients who were given NAC for locally-advanced esophageal cancer at the National Defense Medical College Hospital between 2009 and 2017. The study included 14 women and 77 men with a median age of 71 (range 43-86) years. Fifteen patients underwent Lap-J prior to NAC (Lap-J group), and 76 patients received NAC without Lap-J (control group). The patient characteristics are shown in Table I. Lap-J was indicated in patients with dysphagia because of stenosis that could not be passed by a standard gastroscopy and nasogastric tube. The contraindications for Lap-J were unsuitability for general anesthesia and gastrointestinal disease. Lap-J was not indicated in patients with adequate oral intake of food or liquid supplements. Chemotherapy was administered twice every four weeks, including cisplatin $80 \mathrm{mg} / \mathrm{m}^{2}$ by intravenous drip infusion for $2 \mathrm{~h}$ on day 1 and 5 -FU $800 \mathrm{mg} / \mathrm{m}^{2}$ by continuous infusion on days 1-5 (1). Nutritional quality was monitored by body weight, body mass index (BMI), total blood protein and albumin concentrations, total lymphocyte count, hemoglobin, prognostic nutritional index (PNI) (4), modified Glasgow prognostic score (mGPS) (5), and psoas muscle index (PI) before and after NAC. The blood tests were measured using biochemical analyzer (LABOSPECT 008, Hitachi High-Technologies, Tokyo, Japan) and blood cell analyzer (XE5000, Sysmex, Hyogo, Japan). Computed tomography (CT) scanning was undergone before and after NAC, in the supine position, from neck to pelvis, with a 64-multidetector CT scanner (Aquilion 64, Toshiba, Tokyo, Japan). The 
PI $\left(\mathrm{cm}^{2} / \mathrm{m}^{2}\right)$ was calculated from CT images and the psoas muscle cross-sectional area at the L3 level normalized against length (6). The mean total observation period was 722 days (95\% confidence interval 565 to 880 ). The study protocol was reviewed and approved by the Institutional Review Board of the National Defense Medical College. Written informed consent was obtained from each patient prior to study inclusion.

Lap-J procedure. Lap-J was performed as previously described (3). In brief, under general anesthesia and after laparoscopic exploration of the peritoneal cavity, the jejunum 20-30 cm distant from the Treitz ligament was pulled out through the umbilical trocar incision. A serosal suture was placed, and a jejunostomy was performed by the Witzel technique using a needle jejunostomy kit (Covidien, Tokyo, Japan). The jejunal loop was gently returned to the abdomen and the feeding tube was drawn through the abdominal wall via the left lower trocar incision. The jejunum was then laparoscopically sutured to the anterior abdominal wall with approximately six sutures.

Statistical analysis. Normally-distributed continuous data were reported as means and standard error (SE). Non-normally distributed continuous data were reported as medians and interquartile range (IQR). Categorical variables were reported as numbers and percentages. Differences in normally-distributed clinical variables were compared using the student t-test. Pearson's chi-square test or Fisher's exact test were used for categorical data. The Mann-Whitney U test was used to analyze non-normally distributed continuous and ranked data, as appropriate. Overall (OS) and disease-free (DFS) survival were estimated by the Kaplan-Meier method. The significance of differences in survival was compared with the log-rank test. The significance of the association of clinical variables and patient outcomes was tested by univariate analysis. Variables with a P-value $<0.10$ were included in a subsequent multivariate (logistic regression or Cox proportional hazards regression) analysis. All tests were two-sided, and P-values $<0.05$ were considered statistically significant. The statistical analysis was performed using JMP statistics version 11.0 (SAS Institute Inc., Carey, NC, USA).

\section{Results}

There were no significant differences in tumor characteristics (location, depth, or nodal involvement) in the 2 groups. Forty-eight patients had no nutritional support during NAC, 26 required nutritional liquid supplements, and two required nasogastric tube feeding. All patients in the Lap-J group were able to stand, walk, and begin enteral nutrition via the jejunostomy on postoperative day 1 . Fourteen of the 15 patients $(93.3 \%)$ did not experience any procedure-related complications. One had subcutaneous abscess necessitating the removal of jejunostomy tube during NAC. NAC began at a median of seven days after Lap-J surgery (range, 3-19 days). No mortalities were associated with Lap-J.

The nutritional characteristics in the two groups before the start of NAC are shown in Table II. Body weight, BMI, PI, serum albumin, and PNI were significantly lower in the Lap-J group in comparison with the control group. Two score
Table I. Clinicopathological characteristics of Lap-J and control patients.

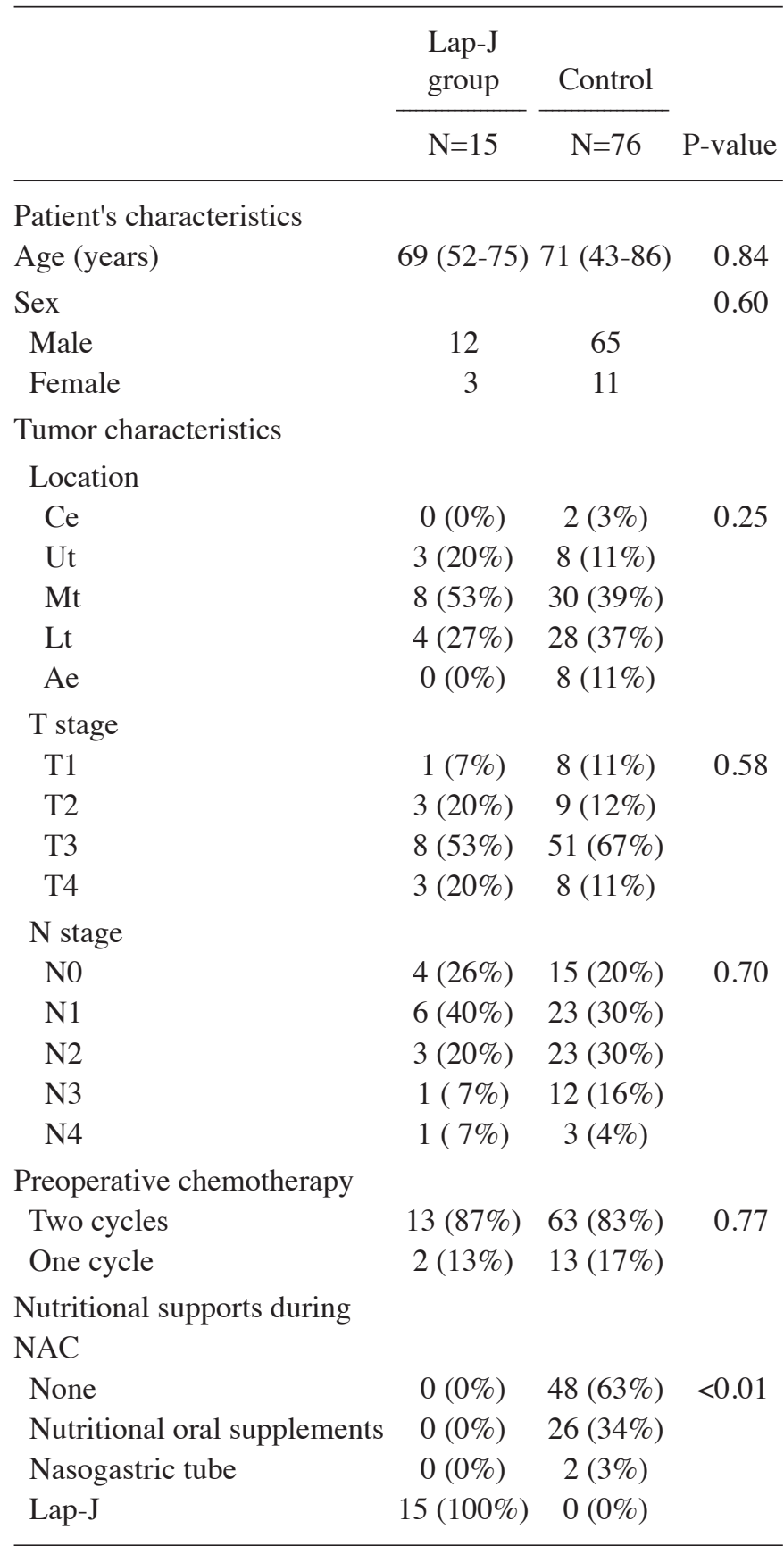

Ce, cervical esophagus; Ut, upper thoracic esophagus; Mt, middle thoracic esophagus; Lt, lower thoracic esophagus; Ae, abdominal esophagus; NAC, neoadjuvant chemotherapy; Lap-J, laparoscopic jejunostomy.

of mGPS occurred more frequently in the Lap-J group than in the control group. Changes in the values of nutritional variables during NAC are shown in Fig. 1. Significant decreases in serum total protein, albumin, and hemoglobin and in PNI occurred during NAC in the control group but not in the Lap-J group. Serum albumin was significantly higher in the Lap-J group after NAC than in the control group. The number of patients with a 2 score of mGPS in the control group increased after NAC, but decreased in the Lap-J group (Fig. 2). There 

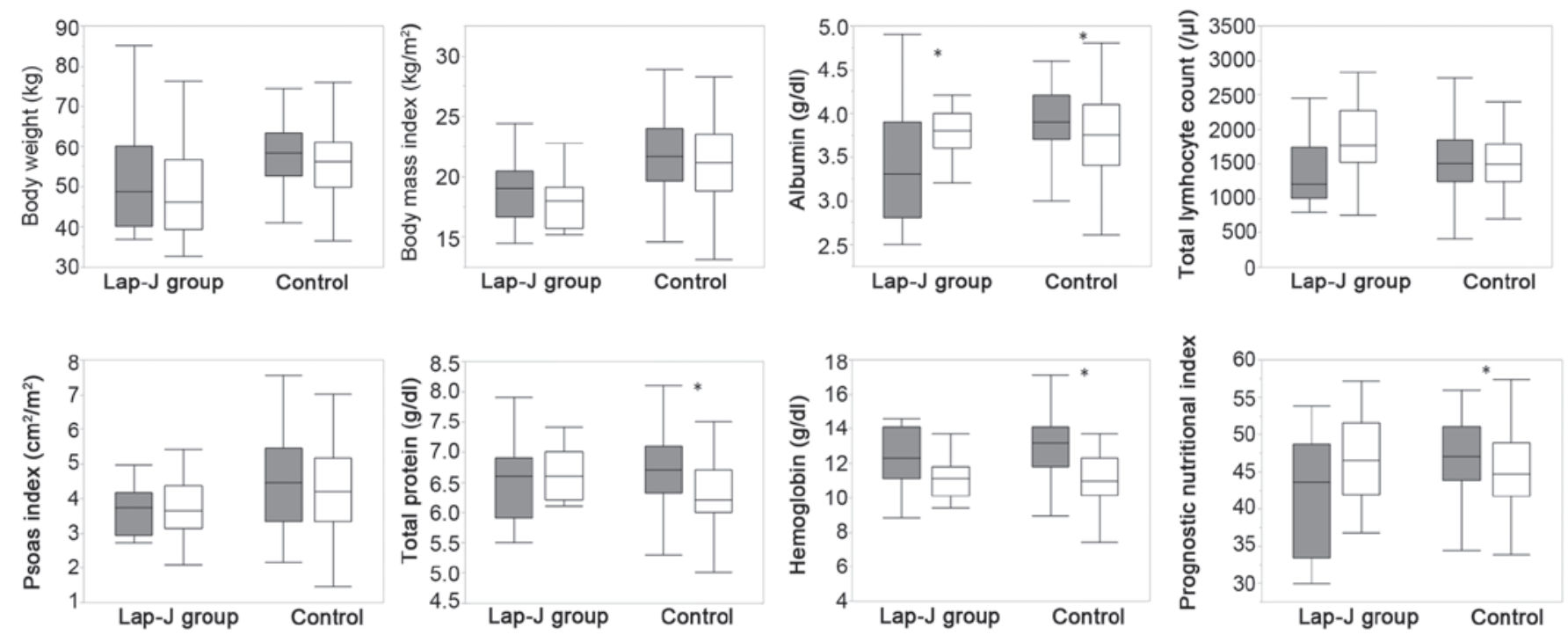

Figure 1. Changes in nutritional variables during NAC. The dark and white bars indicated the values of each nutritional parameter before and after NAC, respectively. Serum total protein, albumin, hemoglobin and PNI significantly decreased during NAC in the control group, but did not decrease in the Lap-J group. Serum albumin level significantly increased during NAC in the Lap-J group. " $\mathrm{P}<0.05$ vs. after NAC. NAC, neoadjuvant chemotherapy; Lap-J, laparoscopic jejunostomy; PNI, prognostic nutritional index.
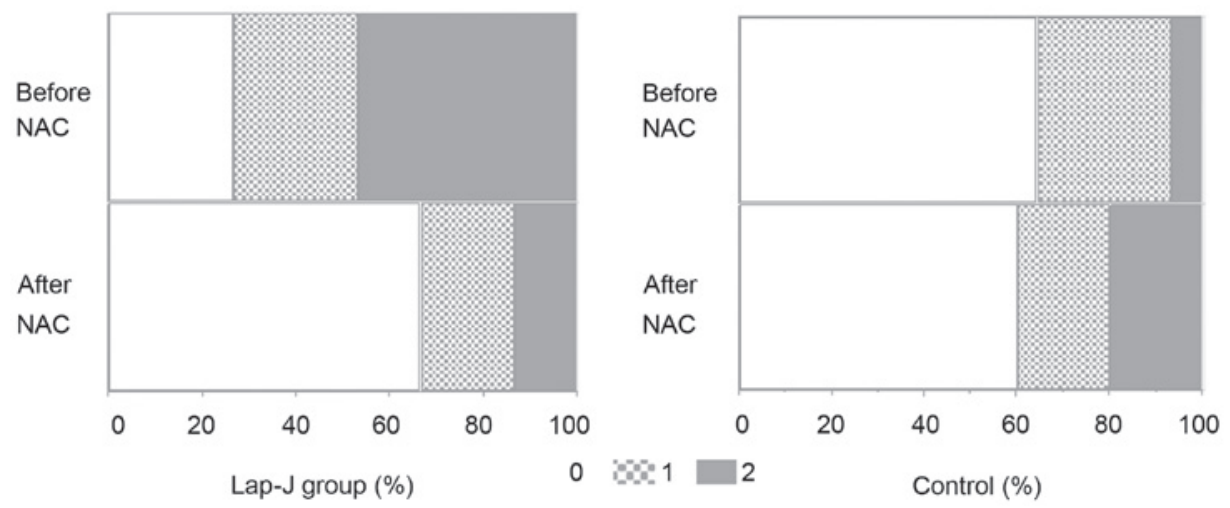

Figure 2. mGPS before and after NAC. The percentage of patients with a 2 score of mGPS in the control group increased after NAC, and decreased in the Lap-J group. mGPS, modified Glasgow prognostic score; NAC, neoadjuvant chemotherapy; Lap-J, laparoscopic jejunostomy.
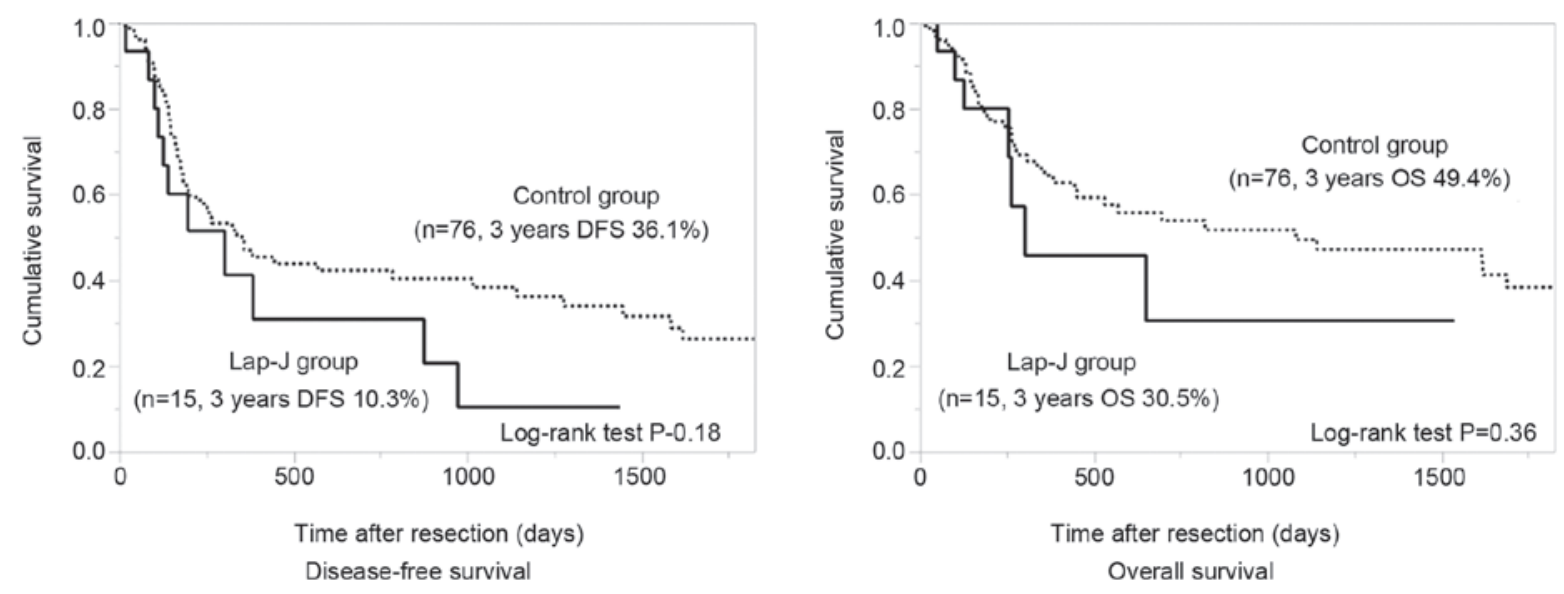

Figure 3. DFS and OS rates in the Lap-J and control groups. There were no significant differences in DFS and OS of the two groups. Lap-J, laparoscopic jejunostomy; DFS, disease-free survival; OS, overall survival.

were no differences in the occurrence of postoperative complications, i.e., anastomotic leakage (Lap-J 27\% vs. control 24\%) and pneumonia (Lap-J 27\% vs. control 38\%). The differences in DFS and OS of the two groups were not significant (Fig. 3). 
Table II. Nutritional variables prior to neoadjuvant chemotherapy.

\begin{tabular}{lccr}
\hline & $\begin{array}{l}\text { Lap-J } \\
\text { group }\end{array}$ & Control & P-value \\
\hline Age, years & $69(52-75)$ & $71(43-86)$ & 0.84 \\
Sex male/female & $12 / 3$ & $65 / 11$ & 0.60 \\
Body weight $(\mathrm{kg})$ & $50.5 \pm 13.0$ & $57.9 \pm 8.4$ & $<0.01$ \\
BMI $\left(\mathrm{kg} / \mathrm{m}^{2}\right)$ & $19.4 \pm 4.3$ & $21.9 \pm 3.1$ & $<0.05$ \\
PMI $\left(\mathrm{cm}^{2} / \mathrm{m}^{2}\right)$ & $3.7 \pm 0.7$ & $4.6 \pm 1.4$ & $<0.05$ \\
Total protein $(\mathrm{g} / \mathrm{dl})$ & $6.5 \pm 0.7$ & $6.7 \pm 0.5$ & 0.16 \\
Albumin $(\mathrm{g} / \mathrm{dl})$ & $3.5 \pm 0.7$ & $3.9 \pm 0.4$ & $<0.01$ \\
Total lymphocyte count $(/ \mu 1)$ & $1398 \pm 521$ & $1572 \pm 509$ & 0.23 \\
Hemoglobin $(\mathrm{g} / \mathrm{dl})$ & $12.1 \pm 1.9$ & $13.0 \pm 1.7$ & 0.06 \\
Prognostic nutritional index & $41.6 \pm 8.0$ & $46.9 \pm 4.9$ & $<0.01$ \\
Modified Glasgow prognostic & & & \\
score & & & \\
0 & $4(26.7 \%)$ & $49(64.5 \%)$ & \\
1 & $4(26.7 \%)$ & $22(28.9 \%)$ & $<0.01$ \\
2 & $7(46.7 \%)$ & $5(6.6 \%)$ & \\
\hline
\end{tabular}

Lap-J, laparoscopic jejunostomy; BMI, body mass index; PMI, psoas muscle index; NAC, neoadjuvant chemotherapy.

\section{Discussion}

Despite progress in perioperative management and operative procedures, esophagectomy for esophageal cancer is still a highly-invasive procedure associated with serious postoperative complications, including pneumonia and anastomotic leakage $(7,8)$. Combination chemotherapy for esophageal carcinoma has improved local tumor control and reduced the incidence of distant treatment failure after surgery. However, esophageal cancer is still one of the most refractory cancers (1). Poor nutrition increases the risk of poor short- and long-term outcomes in patients with esophageal cancer $(9,10)$. Preoperative chemotherapy in itself induces malnutrition because of treatment-related anorexia and nausea. Patients with advanced esophageal cancer frequently experience dysphagia from stenosis caused by the tumor. Appropriate nutritional support during NAC is essential for the improvement of surgical outcomes in patients with advanced esophageal cancer.

Among the nutritional interventions that are available during preoperative therapy, enteral feeding by a nasogastric tube is the easiest and least invasive intervention. However, in addition to discomfort, nasogastric tubes are subject to accidental extubation, aspiration risk, and the need of hospitalization, all of which compromise patients' quality of life. Temporary endoscopic stent placement provides instant resolution of dysphagia, but often causes clinically significant complications such as stent migration and esophago-tracheo-bronchial fistulas $(11,12)$. The use of esophageal stents in the neoadjuvant setting may lead to complications that can compromise the opportunity for curative surgery in a small proportion of patients (13). Percutaneous endoscopic gastrostomy has been described as an option for provision of nutrition (14), but it might interfere with the subsequent use of a gastric conduit for reconstruction. Feeding jejunostomies are effective and reasonable options for providing perioperative nutritional support, and prolonged tube feeding can be continued after radical esophagectomy (15).

In this study, Lap-J feeding effectively maintained serum total protein, albumin, and hemoglobin, and the PNI during NAC compared with the control group. In addition to maintaining nutritional status, Lap-J contributed to improved quality of life in patients with obstructing esophageal cancer because they could live at home except for receiving chemotherapy infusions. The study did not demonstrate any benefits of Lap-J associated with infectious complications, hospital stay, or long-term outcome after radical esophagectomy compared with the control group. That might have been a consequence of the small number of patients undergoing Lap-J and the presence of more advanced disease in patients with obstructing esophageal tumors than in those patients with non-obstructing esophageal tumors. The results support the conduct of a randomized controlled trial with a larger patient population to evaluate the impact of Lap-J on the perioperative management of obstructing esophageal cancer. In addition, the superiority of Lap-J over conventional nutritional supports such as nasogastric tube and temporary stent should be clarified in near future to demonstrate the efficacy of Lap-J.

Lap-J has several advantages over conventional open procedure. Firstly, lap-J can explore the whole abdominal cavity, which may detect small liver and peritoneal metastases (16). Secondly, Lap-J possesses the minimal invasive procedure as compared to open procedure, which makes it possible to start the following therapy $(17,18)$.

In conclusion, we recommend Lap-J for safe and effective perioperative nutritional support during NAC in patients with obstructing esophageal cancer. However, the survival advantage of Lap-J during NAC should be clarified in the prospective randomized study with sufficient number of patients.

\section{Acknowledgements}

Not applicable.

\section{Funding}

No funding was received.

\section{Availability of data and materials}

All data used and/or analyzed during this published article are available from the corresponding author on reasonable request.

\section{Authors' contributions}

$\mathrm{KN}, \mathrm{HT}$ and HU collaborated in the conception and design of the study. KN, HN, MH, NI, SN and $\mathrm{HH}$ acquired the data. $\mathrm{KN}$, $\mathrm{HT}, \mathrm{SH}, \mathrm{SA}, \mathrm{KH}$ and HU performed data analysis and interpretation. All authors were involved in writing the manuscript. All authors read and approved the final manuscript.

\section{Ethics approval and consent to participate}

Not applicable. 


\section{Patient consent for publication}

The patient provided written informed consent for the publication of their data.

\section{Competing interests}

The authors declare that they have no competing interests.

\section{References}

1. Ando N, Kato H, Igaki H, Shinoda M, Ozawa S, Shimizu H Nakamura T, YabusakiH,Aoyama N,Kurita A, etal: A randomized trial comparing postoperative adjuvant chemotherapy with cisplatin and 5-fluorouracil versus preoperative chemotherapy for localized advanced squamous cell carcinoma of the thoracic esophagus (JCOG9907). Ann Surg Oncol 19: 68-74, 2012.

2. Sun J, Wang D, Mei Y, Jin H, Zhu K, Liu X, Zhang Q and Yu J: Value of the prognostic nutritional index in advanced gastric cancer treated with preoperative chemotherapy. J Surg Res 209: 37-44, 2017.

3. Tsujimoto $\mathrm{H}$, Hiraki $\mathrm{S}$, Takahata $\mathrm{R}$, Nomura S, Ito $\mathrm{N}$, Kanematsu K, Horiguchi H, Aosasa S, Yamamoto J and Hase K: Laparoscopic jejunostomy for obstructing upper gastrointestinal malignancies. Mol Clin Oncol 3: 1307-1310, 2015.

4. Smale BF, Mullen JL, Buzby GP and Rosato EF: The efficacy of nutritional assessment and support in cancer surgery. Cancer 47: 2375-2381, 1981

5. Proctor MJ, Morrison DS, Talwar D, Balmer SM, Fletcher CD, O'Reilly DS, Foulis AK, Horgan PG and McMillan DC: A comparison of inflammation-based prognostic scores in patients with cancer. A Glasgow Inflammation Outcome Study. Eur J Cancer 47: 2633-2641, 2011.

6. Hiraoka A, Aibiki T, Okudaira T, Toshimori A, Kawamura T, Nakahara H, Suga Y, Azemoto N, Miyata H, Miyamoto Y, et al: Muscle atrophy as pre-sarcopenia in Japanese patients with chronic liver disease: Computed tomography is useful for evaluation. J Gastroenterol 50: 1206-1213, 2015.

7. Tsujimoto H, Takahata R, Nomura S, Yaguchi Y, Kumano I, Matsumoto Y, Yoshida K, Horiguchi H, Hiraki S, Ono S, et al: Video-assisted thoracoscopic surgery for esophageal cancer attenuates postoperative systemic responses and pulmonary complications. Surgery 151: 667-673, 2012.
8. Takeuchi H, Miyata H, Gotoh M, Kitagawa Y, Baba H, Kimura W, Tomita N, Nakagoe T, Shimada M, Sugihara K and Mori M: A risk model for esophagectomy using data of 5354 patients included in a Japanese nationwide web-based database. Ann Surg 260: 259-266, 2014.

9. Mariette C, Taillier G, Van Seuningen I and Triboulet JP: Factors affecting postoperative course and survival after en bloc resection for esophageal carcinoma. Ann Thorac Surg 78: 1177-1183, 2004.

10. Law S, Wong KH, Kwok KF, Chu KM and Wong J: Predictive factors for postoperative pulmonary complications and mortality after esophagectomy for cancer. Ann Surg 240: 791-800, 2004.

11. Langer FB, Schoppmann SF, Prager G, Tomaselli F, Pluschnig U, Hejna M, Schmid R and Zacherl J: Temporary placement of self-expanding oesophageal stents as bridging for neo-adjuvant therapy. Ann Surg Oncol 17: 470-475, 2010.

12. Jones CM and Griffiths EA: Should oesophageal stents be used before neo-adjuvant therapy to treat dysphagia in patients awaiting oesophagectomy? Best evidence topic (BET). Int J Surg 12: 1172-1180, 2014.

13. Bower M, Jones W, Vessels B, Scoggins C and Martin R: Nutritional support with endoluminal stenting during neoadjuvant therapy for esophageal malignancy. Ann Surg Oncol 16: 3161-3168, 2009.

14. Bhatti AB, Rizvi FH, Waheed A, Raza SH, Syed AA, Khattak S and Aasim Yusuf M: Does prior percutaneous endoscopic gastrostomy alter post-operative outcome after esophagectomy. World J Surg 39: 441-445, 2015.

15. Gupta V: Benefits versus risks: A prospective audit. Feeding jejunostomy during esophagectomy. World J Surg 33: 1432-1438, 2009.

16. Grondona P, Andreani SM, Barr N and Singh KK: Laparoscopic feeding jejunostomy technique as part of staging laparoscopy. Surg Laparosc Endosc Percutan Tech 15: 263-266, 2005.

17. Han-Geurts IJ, Lim A, Stijnen T and Bonjer HJ: Laparoscopic feeding jejunostomy: A systematic review. Surg Endosc 19: 951-957, 2005.

18. Siow SL, Mahendran HA, Wong CM, Milaksh NK and Nyunt M: Laparoscopic T-tube feeding jejunostomy as an adjunct to staging laparoscopy for upper gastrointestinal malignancies: The technique and review of outcomes. BMC Surg 17: 25, 2017. 